Article available at http://www.parasite-journal.org or nttp://dx.dol.org/10.1051/parasite/1999063243

\title{
Ultrastructural localization OF SM15 AND SM25, TWO MAJOR TEGUMENTAL ADULT WORM ANTIGENS OF SCHISTOSOMA MANSONI
}

\author{
ABATH F.G.C.*, HIRST E.M.A.**, HAGAN P.** \& SIMPSON A.J.G.***
}

Summary :

Sm 15 and Sm25 are two of the principal tegumental antigens recognized by antibodies from mice protectively vaccinated with adult worm tegumental membranes and may therefore be potential vaccine candidate antigens. Using antibodies affinity purified from anti-tegumental membrane anti-sera, and antibodies raised against the recombinant antigens, Sm 15 and Sm25 were shown to be located specifically in the tegument of adult worms being distributed throughout the syncitium but not associated with the outer membrane.

KEY WORDS : Schistosoma mansoni, tegumental antigens, immunolocalization, Sm25, Sm15.
Résumé : LOCAlisATION EN ULTRASTRUCTURE DE DEUX DES PRINCIPAUX ANTIGÈnES TÉGUMENTAIRES DE SCHISTOSOMA MANSON, SM15 ET SM25

Sm 15 et Sm25 sont deux parmi les principaux antigènes tégumentaires reconnus par des anticorps produits par des souris qui ont été vaccinées avec des membranes tégumentaires de Schistosoma mansoni adultes et qui sont, en conséquence, des candidats potentiels à un vaccin spécifique contre ce parasite. Des anticorps purifiés par affinité à partir de sérum anti-membrane tégumentaire, ainsi que des anticorps obtenus contre des antigènes recombinants, ont été employés pour

l'immunolocalisation de Sm 15 et de Sm25. Les résultats ont montré que ces antigènes se trouvent spécifiquement dans le tégument des vers adultes, distribués dans tout le syncitium mais n'étant pas cependant associés à la membrane externe.

MOTS CLÉS : Schistosoma mansoni, antigènes tégumentaires, immunolocalisation, Sm25, Sm 15

$25 \mathrm{kDa}$ antigen (Sm25) (Omer Ali et al., 1991; El-Sherbeini et al., 1991) and the $15 \mathrm{kDa}$ antigen (Sm15) (Abath et al., 1993) have been cloned and sequenced and the mature gene product analyzed in some detail (Karcz et al., 1988; Pearce et al., 1991; Abath et al., 1994).

We have investigated the distribution of Sm15 and Sm25 within adult worms by immuno-gold electron microscopy. Both Sm15 and Sm25 are distributed throughout the adult tegument but are apparently not exposed on adult worm surfaces.

\section{MATERIALS AND METHODS}

\section{PARASITES}

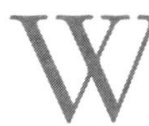

Torms from a Puerto Rican strain of Schistosoma mansoni maintained in the laboratory were obtained from six-week infected hamsters by perfusion from the hepatic portal system (Smithers \& Terry, 1965).

\section{ANTISERA}

Rabbit anti-tegumental membrane antibodies were raised by immunization with tegumental membrane proteins solubilized in SDS (sodium dodecyl sulphate), as described by Simpson et al. (1990). The experiments of immunolocalization were undertaken with sera from 
rabbits immunized with purified recombinant Sm25 (Omer Ali et al., 1991) and affinity purified antibodies, specific for Sm15 (Abath et al., 1994).

\section{IMMUNO-GOLD LABELLING OF ADULT WORM SECTIONS}

Adult worms of S. mansoni were fixed either in $0.1 \%$ high vacuum distilled glutaraldehyde for $30 \mathrm{~min}$ at $4^{\circ} \mathrm{C}$, and then quenched in $1 \%$ ammonium chloride, or $2 \%$ paraformaldehyde/ $1 \%$ acrolein for $30 \mathrm{~min}$ at room temperature. Samples were then washed in PBS pH 7.2, dehydrated at progressively lowered temperatures and embedded in Lowicryl $\mathrm{K} 4 \mathrm{M}$ at $-35^{\circ} \mathrm{C}$ (Roth et al., 1981). Sections $60 \mathrm{~nm}$ thick were cut on a Reichert Ultracut Microtome and placed onto formvar/carbon coated 200 mesh gold grids. Sections were pre-blocked with $10 \%$ non fat dry milk in TBS (tris buffered saline) $\mathrm{pH} 8.2$ for $30 \mathrm{~min}$ at room temperature. Grids were then transferred to antibodies specific for Sm15 diluted 1:100 in TBS containing $1 \%$ BSA and $0.25 \%$ Tween 20 (TBS/BSA/Tween), and incubated overnight at $4{ }^{\circ} \mathrm{C}$. Control sections were treated with the same dilution of normal rabbit serum. The grids were washed for one hour at room temperature with TBS/BSA/Tween, then incubated at room temperature with goat anti-rabbit IgG, conjugated to $10 \mathrm{~nm}$ gold particles (diluted 1:50 in TBS/BSA/Tween). After two hours of incubation, the grids were rinsed in PBS-BSA-Tween, followed by distilled water. The sections were dried, stained with uranyl acetate and viewed in a Phillips 300 electron microscope. Studies involving the localization of $\mathrm{Sm} 25$ used rabbit antibodies raised against a Sm25/glutathione-S-transferase (GST) fusion protein produced using the pGEX-1N expression vector. Negative controls utilized rabbit anti-GST antibodies. Evaluation of titers of the two antisera by ELISA, using GST (enzyme linked immunosorbent assay) indicated that the negative control serum had a fourfold higher titer of antibody against GST than the anti-fusion protein serum. The sections used for the localization of Sm25 were processed exactly as for studies with $\mathrm{Sm} 15$ except that only $0.1 \%$ glutaraldehyde was used as a fixative. In the case of $\operatorname{Sm} 25$ an estimation of the distribution of gold particles was performed by counting the total gold particles in two distinct areas of the tegument and calculating the proportion for those associated to the discoid bodies.

\section{RESULTS}

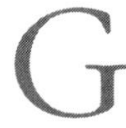
old immunolabelling of adult worm sections using affinity purified antibodies specific for Sm15 and antibodies raised against recombinant Sm25: we have previously demonstrated by immunofluorescence that affinity purified antibodies, which recognize $\mathrm{Sm} 15$, showed a positive and specific reaction with the tegument of adult worms (Abath et al., 1994). The immunolabelling for electron microscopy with antibodies specific for $\mathrm{Sm} 15$ was performed with different fixative regimens. In figure 1 , samples $\mathrm{A}$ and B were fixed with glutaraldehyde, whereas samples C and $\mathrm{D}$ were fixed with paraformaldehyde/acrolein. Although the labelling was not intense (Fig. 1B), it was associated with the tegument, possibly with some preference for the discoid (elongate) bodies. Figure 1D shows tegumental localization of the labelling, although structural preservation is poor. The gold particles were not associated with the tegumental outer membrane or the muscle.

Immunofluorescence using antibodies raised against a Sm 25 - $\beta$-galactosidase protein have been previously used to demonstrate that $\mathrm{Sm} 25$ is expressed only within the tegument of adult worms (Knight et al., 1989). Here antibodies against a Sm25-GST recombinant were used to study the distribution of the antigen at the ultrastructural level (Fig. 2). The antibody exhibited a generalized binding throughout the tegument often, but not exclusively, associated with the discoid bodies. Analysis of two distinct fields demonstrated that $39 \%$ of the gold particles were associated with these structures. There was no evidence for the association of the antigen with the spines, muscle layer or the tegumental outer membrane. The control serum exhibited an extremely low level of binding within the tegument, less than $10 \%$ of that exhibited by the positive serum despite its much higher titer against GST demonstrating the specificity of the binding exhibited by the antirecombinant Sm25 antibodies.

\section{DISCUSSION}

S $\mathrm{m} 15$ and Sm25 are major antigens recognized by antibodies from mice vaccinated with isolated tegumental membranes (Smithers et al., 1989; Smithers et al., 1990). In addition, evidence from other experimental systems is consistent with $\mathrm{Sm} 25$ being a key target antigen of host protective humoral immune responses (Wright et al., 1988; El-Sherbeini et al., 1990).

The membrane fraction of adult worms used for vaccination studies was isolated by the induced shedding of tegumental components by incubation of live adult worms in phosphate buffered saline (Simpson et al., 1981). This preparation contains membranous components of the tegumental syncitium, including mitochondria, discoid and membranous bodies, in addition to the tegumental outer membrane itself. Both Sm15 and Sm25 have been shown to behave as integral membrane proteins as judged by their quantitative 

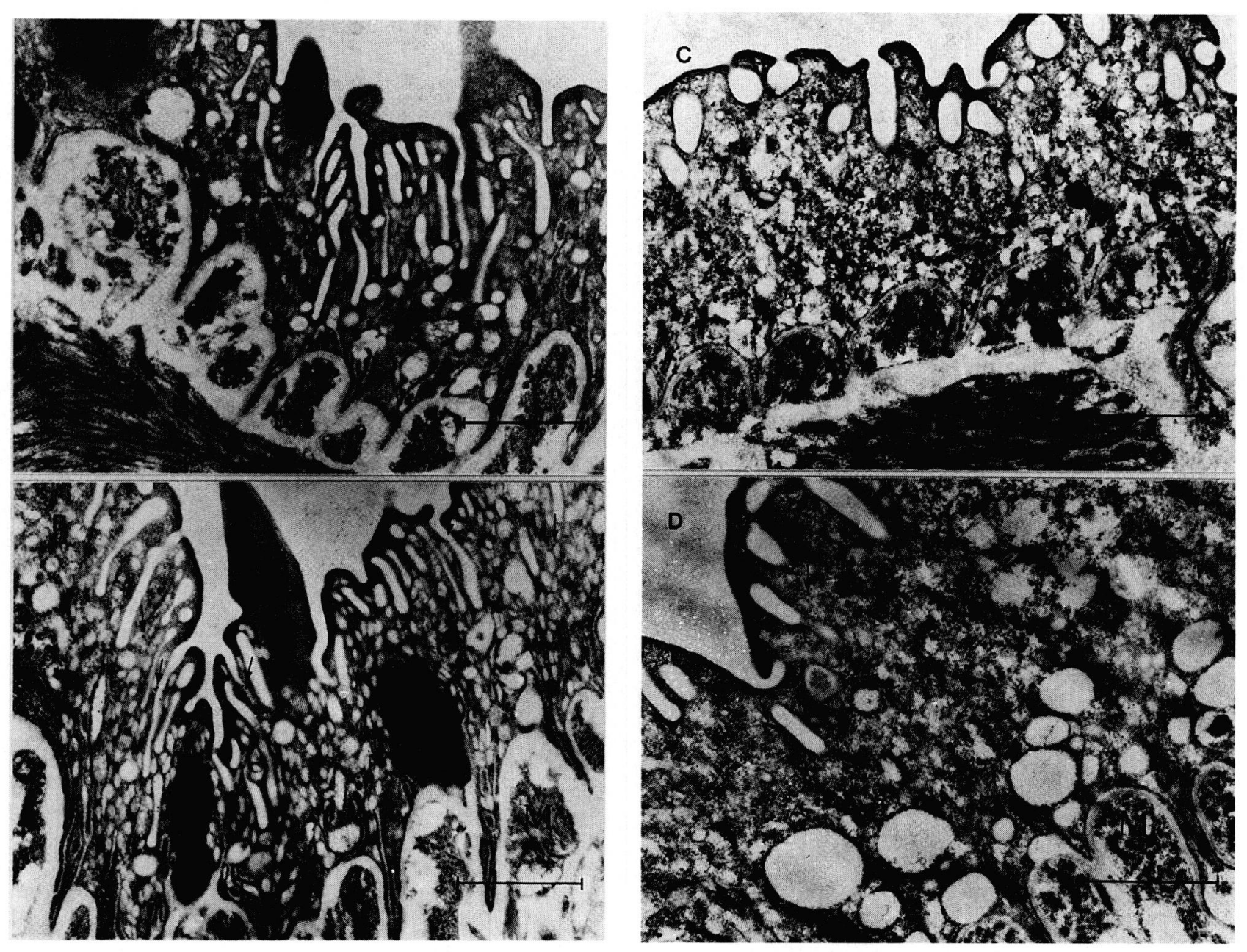

Fig. 1. - Immunoelectron micrographs demonstrating Sm15 in the tegument of adult $S$. mansoni. Gold labelling (arrows) can be observed on the matrix of the syncitial tegumental cytoplasm. In A and B the specimens were fixed with glutaraldehyde while in C and D they were fixed with paraformaldehyde/acrolein. Micrographs A and C show the reactivity of normal rabbit serum. Micrographs B and D show the reactivity of affinity purified antibodies specific for $S \mathrm{~m} 15$. The bars represent $1 \mu \mathrm{m}$. S, spine; M, muscle bundle.

partitioning into the detergent phase upon TX-114 fractionation and are detected in purified tegumental membranes of adult male and female worms (OmerAli et al., 1991; Abath et al., 1994). However, the predicted amino acid sequence of $\mathrm{Sm} 15$ is highly hydrophilic (in contrast to Sm25) with no evidence of hydrophobic domains characteristic of either a transmembrane anchor or the binding of a GPI anchor. Indeed the sequence is uniformly highly acidic which is consistent with the electrophoretic mobility of the antigen in two dimensional gels (Smithers et al., 1990). The basis of its tight membrane association remains to be established, although it is possible that it interacts with membrane molecules (Abath \& Werkhauser, 1996). As highly purified tegumental surface membranes isolated by adsorption to poly-lysine beads contain Sm25 as their dominant component (Payares \& Evans, 1987), the possibility exists for the interaction of Sm15 with Sm25. In addition, experiments involving trypsin digestion showed that during a one hour incubation of live adult worms a substantial portion of the extractable Sm25 was available to the externally applied enzyme. From this it was concluded that the antigen is exposed on the adult parasite surface (Pearce et al., 1991).

The data derived from ultrastructural localization studies failed to demonstrate the presence of either Sm15 or $\mathrm{Sm} 25$ at the parasite surface or indeed associated with the outer membrane of the tegument. Both antigens had a similar distribution being specifically associated with and distributed throughout the tegument, with a tendency for both of them to be associated with the discoid bodies. Although the majority of the particles in both cases are not clearly associated with identifiable membrane structures within the tegument, their quantitative isolation in membrane pellets and distribution in TX-114 phase partitioning indicates that the proteins do not exist in a soluble form. The discoid 


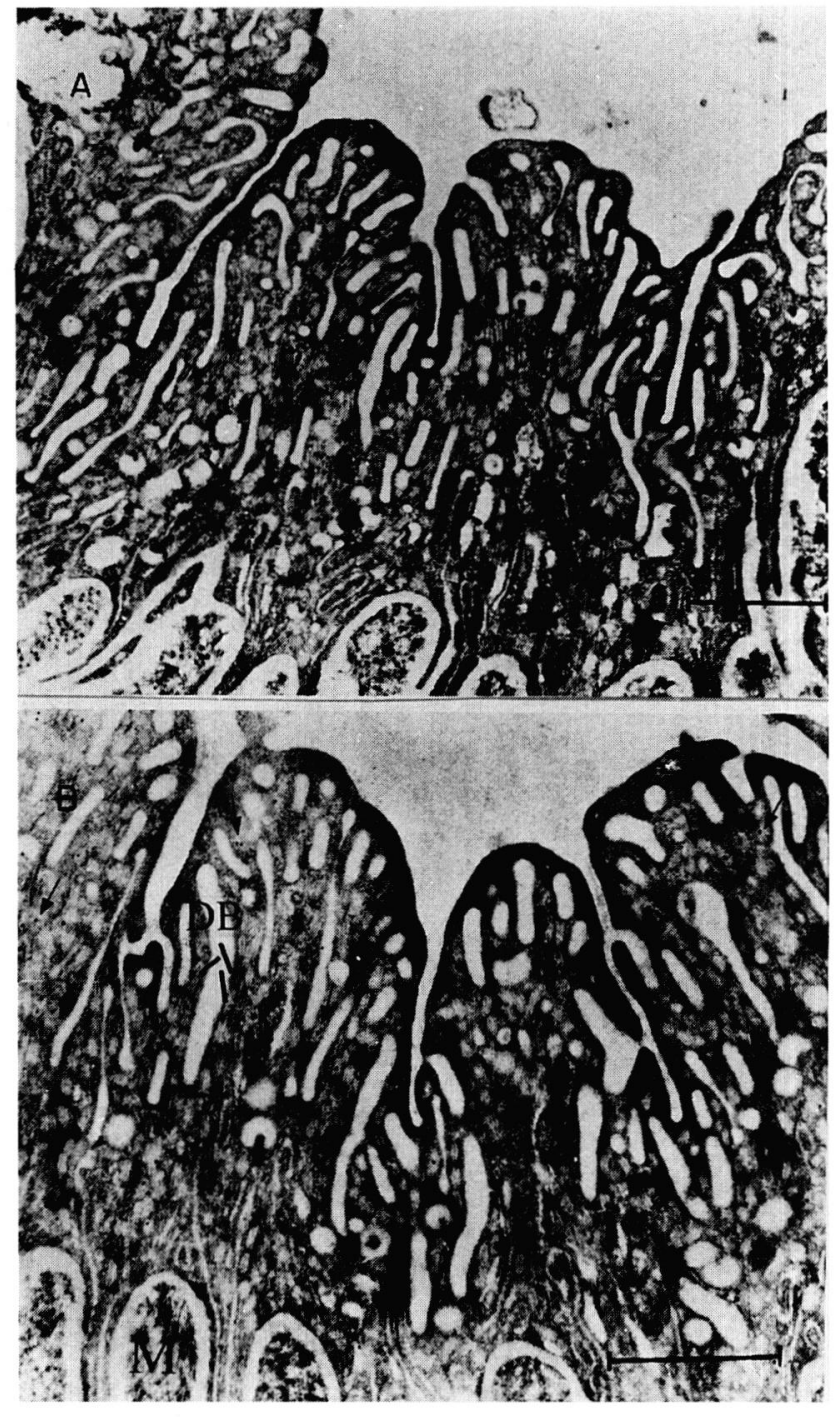

Fig. 2. - Immunoelectron micrographs demonstrating $\mathrm{Sm} 25$ in the tegument of adult $S$. mansoni. Gold labelling (arrows) can be observed on the matrix of the syncitial tegumental cytoplasm, and in association with discoid bodies (some are indicated by lines). The specimens were fixed with glutaraldehyde. Micrograph A show the low reactivity of anti-GST anti-serum. Micrograph B shows the reactivity of rabbit antibodies raised against a Sm25-GST fusion protein. The bars represent $1 \mu \mathrm{m}$. S, spine; $\mathrm{M}$, muscle bundle; $\mathrm{DB}$, discoid bodies.

bodies have been suggested to be involved in biogenesis of the surface bilayers and maintenance of the integrity of the tegument (McLaren, 1980; Zhou et al., 1990). Interestingly, schistosome paramyosin, a protective cytoskeletal protein, has a predominant localization in discoid bodies (Matsumoto et al., 1988), what could suggest that the discoid bodies may also be relevant for the biogenesis of the tegumental cytoskeleton. On the other hand, other tegumental proteins are typically present in discoid bodies and both lipid bilayers that cover the tegumental surface (Jiang et al., 1996; Zhou et al., 1990). The divergence of the biochemical and ultrastructural data concerning the localization of Sm15 and Sm25 necessitates further investigation. It is possible that surface associated molecules are present but they are not conserved by the fixation processes used. It should also be noted that the adult tegument is a dynamic structure highly sensitive to its environment and responds to non ideal conditions by the shedding of membranous fragments and blebs. Certainly the results here indicate a complex and dynamic interaction between the tegumental molecules and demand further investigation.

\section{ACKNOWLEDGEMENTS}

The work was partially funded by FACEPE (Fundacao de amparao a ciencia e tecnologia de Pernambuco) and CNPq (Conselho Nacional de Pesquisa).

\section{REFERENCES}

Abath F.G.C., Hagan P., Jeffs S.A., Schechter I., MeaDows H.M., Holder A.A. \& Simpson A.J.G. Structure of the gene encoding a Schistosoma mansoni putative tegumental antigen precursor. Molecular and Biochemical Parasitology, 1993, 60, 81-92.

Abath F.G.C., Hagan P., JefFs S.A. \& Simpson A.J.G. Partial characterization and kinetics of expression of Sm15, a Schistosoma mansoni tegumental antigen. Parasitology Research, 1994, 80, 64-69.

Abath F.G.C. \& Werkhauser R.P. The tegument of Schistosoma mansoni: functional and immunological features. Parasite Immunology, 1996, 18, 15-20.

El-Sherbeini M., Ramadan N., Bostian K.A. \& Knopf P.M. Cloning and sequence analysis of the Schistosoma mansoni membrane glycoprotein antigen gene GP22. Molecular and Biochemical Parasitology, 1991, 49, 83-98.

Jiang J., Skelly P.J., Shoemaker C.B. \& Caulfield J.P. Schistosoma mansoni: the glucose transport protein SGTP4 is present in tegumental multilamellar bodies, discoid bodies, and the surface bilayers. Experimental Parasitology, 1996, 82, 201-210.

Karcz S.R., Barnard B.J. \& Podesta R.B. Biochemical properties of a 24 kilodalton membrane glycoprotein antigen complex from Schistosoma mansoni. Molecular and Biochemical Parasitology, 1988, 31, 163-172.

Knight M., Kelly.C., Rodrigues V., Yi X., Wamachi A., SmiTHERS S.R. \& SimpSON A.J.G. A cDNA clone encoding part of the major 25000-dalton surface membrane antigen of adult Schistosoma mansoni. Parasitology Research, 1989, 75, 280-286.

Matsumoto Y., Perry G., Levine R.J.C, Blanton R, MahMOUD A.A.F. \& AIKAWA M. Paramyosin and actin in schistosomal teguments. Nature, 1988, 333, 76-78.

MCLAREN D.J. The parasite surface in relation to host immunity. Research Studies Press, New York, 1980. 
Omer-Ali P., Jeffs S.A., Meadows H.M., Hollyer T., Owen C.A., Abath F.G.C., Allen R., Hackett F., Smithers S.R. \& Simpson A.J.G. Structure of Sm25, an antigenic integral membrane glycoprotein of adult Schistosoma mansoni. Molecular and Biochemical Parasitology, 1991, 45, 215-222.

PAYARES G. \& EvANS W.H. Surface proteins and antigens of adult Schistosoma mansoni tegumental membranes detached onto poly-lysine coated beads. Molecular and Biochemical Parasitology, 1987, 23, 129-137.

Pearce E.J., Magee A.I., Smithers S.R. \& Simpson A.J.G. Sm25, a major schistosome tegumental glycoprotein is dependent on palmitic acid for membrane attachment. EMBO Journal, 1991, 10, 2741-2746.

Roth J., Bendayan M., Carleman E., Villiger W. \& Garavito M. Enhancement of structural preservation and immunocytochemical staining in low temperature embedded pancreatic tissue. Journal of Histochemistry and Cytochemistry, 1981, 29, 663-671.

Simpson A.J.G., Schryer M.D., Cesari I.M., Evans W.H. \& SMITHERS S.R. Isolation and partial characterisation of the tegumental outer membrane of adult Schistosoma mansoni. Parasitology, 1981, 53, 105-116.

Simpson A.J.G., Hagan P., Hackett F., Ali P.O. \& Smithers S.R. Epitopes expressed on very low Mr Schistosoma mansoni adult tegumental antigens conform to a general pattern of life-cycle cross-reactivity. Parasitology, 1990, 100, 73-81.

Smithers S.R. \& Terry R.J. The infection of laboratory hosts with cercariae of Schistosoma mansoni and the recovery of the adult worms. Parasitology, 1965, 55, 695-700.

Smithers S.R., Hackett F., Ali P.O. \& Simpson A.J.G. Protective immunization of mice against Schistosoma mansoni with purified adult worm surface membranes. Parasite Immunology, 1989, 11, 301-318.

Smithers S.R., Hackett F., Braga V. \& Simpson A.J.G. Immunoblotting identifies additional antigens recognised by mice protectively vaccinated with adult Schistosoma mansoni tegumental membranes. Parasitology Research, 1990, 76, 454-456.

Wright M.D., Rogers M.V., Davern K.M. \& Mitchell G.F. Schistosoma mansoni antigens differentially recognized by resistant WEHI 129/J mice. Infection and Immunity, 1988, 56, 2948-2952.

Zhou Y., Karcz S.R., Podesta R.B. \& Clarke M.W. Localization of a 24-kilodalton glycoprotein in adult Schistosoma mansoni using immunfluorescence and immunoelectron microscopy. Journal of Parasitology, 1990, 76, 601-605.

Reçu le 8 décembre 1998 Accepté le 16 avril 1999 